\title{
SÍNTOMAS NO MOTORES EN PACIENTES CON ENFERMEDAD DE PARKINSON
}

\section{HOSPITAL DE SAN JOSÉ, BOGOTÁ, DC COLOMBIA}

Eduardo Palacios Sánchez MD*, Carlos Javier Perdomo Rivera MD**, Mauricio Patiño Rodríguez MD***

\section{Resumen}

Introducción: la enfermedad de Parkinson (EP) es la segunda afección neurodegenerativa en frecuencia con una prevalencia en Colombia de 4.7 por mil habitantes. Los síntomas no motores (SNM) hacen parte del espectro ya que generan un aumento de la morbilidad e impactan la calidad de vida. Objetivo general: describir la frecuencia de SNM en pacientes con EP que asisten a la consulta externa de neurología en el Hospital de San José de Bogotá DC. Métodos: serie de adultos que consultaron entre abril y septiembre de 2011 cumpliendo criterios diagnósticos de EP según UK Brain Data Bank. Se utilizó el cuestionario de SNM para EP, $P D N M S$ quest, validado en español. Resultados: se encuestaron 55 pacientes con edad promedio de 68 años (DE 9.8), hombres $61.8 \%$. EI 51\%, con un tiempo de evolución menor o igual a cinco años. EI 85.5\% tenían una puntuación en escala de Hoehn y Yahr entre I-II. Los SNM más frecuentes son urgencia urinaria $76.4 \%$, tristeza $74.5 \%$, sensación desagradable en las piernas durante la noche o necesidad de moverlas $67.2 \%$ y problemas para recordar cosas $61.8 \%$. Conclusiones: Ios SNM son frecuentes en nuestra población siendo el sistema urinario es el más comprometido. Se debe indagar sobre estos para brindar un manejo integral al paciente. Se requieren más estudios en nuestra población que evalúen el impacto sobre la calidad de vida.

Palabras clave: Parkinson, síntomas no motores, PD NMS quest, cuestionario.

Abreviaturas: EP, enfermedad de Parkison; SNM, síntomas no motores.

\section{NON-MOTOR SYMPTOMS IN PARKINSON DISEASE PATIENTS hOSPITAL DE SAN JOSÉ, BOGOTÁ DC. COLOMBIA}

\section{Abstract}

Introduction: Parkinson's disease (PD) is the second most common neurodegenerative disorder with a prevalence of 4.7 per 1000 people in Colombia. Non-motor symptoms (NMS) are included in the spectrum for they lead to increased morbility and impaired quality of life. Overall Objective: to describe the frequency of NMS in patients diagnosed with PD who attend the neurology outpatient clinic at Hospital de San José de Bogotá DC. Methods: series of adults who consulted between April and September 2011 who met the diagnostic criteria for PD according to the UK Brain Data Bank. The PD NMS quest questionnaire validated in Spanish was used. Results: fifty-five patients, men 61.8\%, average age 68 years (SD 9.8) were surveyed. A clinical course equal to or less than 5 years was documented in $51 \%$

Fecha recibido: enero 10 de 2012 - Fecha aceptado: enero 20 de 2012

* Profesor Titular de neurología, Fundación Universitaria Ciencias de la Salud. Jefe del Servicio de neurología, Hospital de San José. Bogotá DC. Colombia.
** Residente IV año de neurología, Fundación Universitaria Ciencias de la Salud, Bogotá DC. Colombia.

*** Residente II de neurología, Fundación Universitaria de Ciencias de la Salud, Bogotá DC. Colombia. 
and $85.5 \%$ were classified between stages I and II according to the Hoehn and Yahr scale. The most common NMS are: urgent urination $\mathbf{7 6 . 4 \%}$, melancholia $\mathbf{7 4 . 5 \%}$, unpleasant sensation in the legs during the night or need to move the legs $67.2 \%$ and having trouble remembering things $61.8 \%$. Conclusions: NMS are often found in our population. The urinary tract is most commonly involved. Patient should be asked about NMS in order to offer a comprehensive management. Further studies on our population evaluating quality of life are required.

Key words: Parkinson, non-motor symptoms, PD NMS quest, questionnaire.

\section{Introducción}

La EP descrita hace más 200 años por James Parkinson en el ensayo sobre la parálisis agitante ${ }^{1,2}$ es la segunda patología neurodegenerativa más frecuente. Afecta del 1 al 1,43\% de la población mundial. ${ }^{3}$ En Colombia, el estudio neuroepidemiológico nacional (EPINEURO), determinó una prevalencia de 4,7 por cada mil habitantes. ${ }^{4}$ Se caracteriza por bradicinesia, rigidez y temblor en reposo, lo que se asocia con SNM como alteraciones del sueño, incontinencia urinaria, estreñimiento, disfagia, sialorrea e incluso síntomas psicóticos. ${ }^{1,5,6}$ Estos últimos tienen impacto negativo en la calidad de vida de los pacientes que la padecen, generando aumento de la morbilidad. Algunos juegan un papel importante como posibles predictores de la enfermedad, siendo responsables de la institucionalización e incremento del costo de su cuidado. ${ }^{1,-15}$
En la EP hay una degeneración progresiva de las neuronas dopaminérgicas de la sustancia nigra pars compacta, que constituye la base de los síntomas motores. Sin embargo, los sustratos neuroanatómicos y neuroquímicos de la mayoría de los SNM aún son desconocidos. ${ }^{15}$ La degeneración de sistemas celulares no dopaminérgicos preceden al desarrollo de la mayoría de los SNM, incluyendo el sistema noradrenérgico del locus coeruleus, el serotoninérgico del rafe, los colinérgicos del núcleo basal de Meynert y otros núcleos colinérgicos del tronco encefálico, como el tegmental pedunculopontino. ${ }^{15}$ Una explicación de la fisiopatología de los SNM es la teoría de Braak $^{5,6,15}$, según la cual se desarrolla un proceso patológico progresivo a lo largo del sistema nervioso en sentido caudorrostral, con seis estadios (Tabla 1), lo que explica que los SNM iniciales pueden ser predictivos de la enfermedad, con la subsiguiente aparición

\begin{tabular}{|c|c|c|}
\hline Estadio & Sustrato anatómico & Manifestaciones clínicas \\
\hline 1 & $\begin{array}{l}\text { Degeneraciones del bulbo olfatorio, núcleo } \\
\text { olfatorio anterior y el motor dorsal del vago }\end{array}$ & $\begin{array}{l}\text { Manifestaciones premotoras: hiposmia } \\
\text { y síntomas autonómicos }\end{array}$ \\
\hline 2 & $\begin{array}{l}\text { Degeneración del tegmento pontino (núcleos del } \\
\text { rafe) y del núcleo tegmental pedunculopontino }\end{array}$ & $\begin{array}{l}\text { Síntomas premotores: trastornos } \\
\text { del sueño }\end{array}$ \\
\hline 3 & Afección del mesencéfalo & Síntomas motores \\
\hline 4 & Afección del prosencéfalo basal & Síntomas motores \\
\hline 5 & $\begin{array}{l}\text { Compromiso de la neocorteza: corteza } \\
\text { prefrontal }\end{array}$ & $\begin{array}{l}\text { Síntomas no motores: alteración } \\
\text { cognitiva }\end{array}$ \\
\hline 6 & $\begin{array}{l}\text { Compromiso de la neocorteza: corteza de } \\
\text { asociación. }\end{array}$ & $\begin{array}{l}\text { Síntomas no motores: alteración } \\
\text { cognitiva }\end{array}$ \\
\hline
\end{tabular}

* Traducida de Braak y colaboradores. ${ }^{5}$ 
de las manifestaciones motoras y después el desarrollo de los SNM tardíos. 3, 6, 15,16

Los SNM tienen una prevalencia que varía desde $21 \%$ al momento del diagnóstico de la EP hasta $88 \%$ después de siete años de evolución. ${ }^{17}$ En muchas oportunidades son subdiagnosticados debido a la inespecificidad de los mismos, el poco tiempo designado para cada consulta, el indagar solo sobre los síntomas motores en el interrogatorio y la atribución a efectos secundarios del tratamiento entre otros, no ofreciéndose suficientes alternativas terapéuticas a los pacientes. ${ }^{14,18}$

Martínez-Martín y colaboradores entre 2007 y 2008 realizaron un amplio estudio en Brasil, India, Italia, Japón, Holanda, Rumania, España, Reino Unido, Estados Unidos y Venezuela, encontrando que en sólo $1,6 \%$ de los pacientes no se documentaron SNM y hubo una correlación positiva con los años de evolución de la enfermedad y con el estadío de Hoehn y Yahr. ${ }^{18-21}$ El mismo estudio evaluó aceptabilidad, confiabilidad, validez y precisión del cuestionario para SNM en EP , PD NMS quest, validado al español. ${ }^{9}$

En nuestra revisión no se encontraron estudios nacionales publicados que determinen la frecuencia de SNM a en la EP. El Hospital de San José atendió una población aproximada de 120 pacientes con EP en el último año, sin que se cuente con registros sobre la frecuencia de SNM que nos alerten para tomar una conducta adecuada en el tratamiento integral y el mejoramiento de la calidad de vida de estos pacientes y sus familias.

El objetivo del estudio es describir la frecuencia de SNM en pacientes con EP que asisten a la consulta externa de neurología en el Hospital de San José.

\section{Materiales y métodos}

Se describe el primer informe de una serie de casos de pacientes atendidos entre abril y septiembre de 2011 en el Hospital de San José. El reporte final se presentará cuando se complete un año de recolección de datos y se obtenga información de calidad de vida de los pacientes. Ingresaron los mayores de 18 años que asistieron a la consulta externa de neurología con diagnóstico de EP de acuerdo con los criterios de UK PD Brain Bank Criteria ${ }^{7,9}$, sin tener en cuenta el grado de severidad de la enfermedad. Se excluyeron quienes no brindaron consentimiento para aportar la información al cuestionario.

Los datos fueron obtenidos a través de entrevista en dos instancias; primero la aplicación del cuestionario, el cual podía ser autodiligenciado o asistido por algún acompañante, interpretando las preguntas bajo la supervisión continua por parte de los investigadores, quienes además determinaron el estadio en la escala de Hoehn Yahr para cada paciente en la valoración clínica. Solo se diligenció un formulario por paciente, independiente del número de consultas en el año. Se tomaron datos de acuerdo con lo referido por el paciente y/o acompañantes, registrando las variables edad, escolaridad, estado civil, actividad laboral, antecedentes patológicos, tiempo de inicio de la enfermedad y farmacoterapia actual para EP.

El cuestionario de SNM validado al español, aceptado y de fácil reproducción se sometió a adaptación cultural con la autorización del autor para ser aplicado en la población de estudio, sin haber sido validado en nuestro país. Consta de 30 preguntas que hacen referencia a la presentación de síntomas en las últimas cuatro semanas a partir del día de la consulta. Se evaluaron diez dominios: tracto gastrointestinal, vía urinaria, dolor, misceláneos, apatía/atención/memoria, depresión/ansiedad/anhedonia, problemas perceptivos/ alucinaciones, función sexual, sueño/fatiga y cardiovascular.

La base de datos se elaboró en Microsoft Office Excel 2007. Para las variables cualitativas se calcularon frecuencias. Las cuantitativas se analizaron por medio de medidas de tendencia central y dispersión en STATA 10. Este trabajo fue aprobado por el Comité de Investigación y Ética de la Fundación Universitaria de Ciencias de la Salud. 


\section{Resultados}

Se reportan datos de 55 pacientes con criterios diagnósticos para $\mathrm{EP}$ a quienes se les aplicó el cuestionario de SNM. El 61.8\% de la población fueron hombres con edad promedio de 68 años (DE 9.8, rango 44 a 94 años) (Tabla 2). Dentro de los antecedentes patológicos en orden de frecuencia fueron hipertensión arterial $36.4 \%$, hipotiroidismo $16.4 \%$ y diabetes mellitus $14.6 \%$. En el $51 \%$ la evolución era menor o igual a cinco años al momento de la encuesta. El 85.5\% tenían una puntuación en la escala de Hoehn y Yahr entre I-II. En relación con el manejo médico para la EP el $83.6 \%$ utilizaba levodopa/carbidopa y $7.2 \%$ recibió monoterapia con agonistas dopaminérgicos. La intervención quirúrgica se registró en el $3.6 \%$ (Tabla 2).

Los SNM registrados con mayor frecuencia distribuidos por dominios fueron tracto urinario con urgencia urinaria $76.4 \%$, depresión/ansiedad, tristeza $74.5 \%$, sueño y fatiga con sensación desagradable en las piernas durante la noche $67.2 \%$, apatía/atención/ memoria con problemas para recordar cosas $61.8 \%$, dolor sin causa aparente $54.6 \%$, gastrointestinal con estreñimiento $47.3 \%$, misceláneos como caídas $38.2 \%$, función sexual con pérdida o aumento de la líbido $43.6 \%$, cardiovascular con sudoración excesiva $43.6 \%$ y alucinaciones al ver u oír cosas que no existían 25.5\% (Tabla 3).

Los SNM en pacientes con menos de cinco años de evolución de la enfermedad en orden de frecuencia fueron: urgencia urinaria $75.4 \%$, sensación desagradable en las piernas por la noche $71.3 \%$ y tristeza o bajo de ánimo $67.3 \%$. En pacientes con más de cinco años en su evolución, los síntomas más frecuentes fueron: sentirse triste $81.4 \%$, urgencia urinaria $77.7 \%$ y nicturia $77.7 \%$.

La presentación de los SNM en su gran mayoría aumentan en relación con el tiempo de evolución, donde predomina el insomnio con un aumento de $23.7 \%$. Por el contrario, hay una reducción en la presentación de sensación desagradable en las piernas por la noche o necesidad de moverlas, dolor sin causa aparente, sudoración excesiva, dificultades para la relación sexual y cambio de peso de $8.4 \%, 12.6 \%, 13 \%, 16.9 \%$ y $9,5 \%$ (Tabla 4$)$.
Tabla 2. Características de la población con EP

Sexo masculino, $\mathrm{n}(\%)$
Edad, años promedio (DE)
rango (años)
Escolaridad, $\mathrm{n}(\%)$
ninguna
primaria
secundaria
universitaria
técnico
Estado civil, $\mathrm{n}(\%)$
casado
viudo
soltero
unión libre
divorciado

Ocupación, activo laboralmente, $\mathrm{n}$

(\%)

Cuidador

Antecedentes personales, $\mathrm{n}(\%)$ hipertensión arterial 20

hipotiroidismo

diabetes mellitus tipo 2

EPOC* 3

SAHOS†

Duración de la EP, promedio(DE)

Tiempo de evolución de (EP), $n$ (\%)

rango (años)

$\leq 5$ años

$(0.5-23)$

6 a 10 años

$>10$ años

Escala de Hoehn Yahr

I-II

III-IV

V

Manejo para Parkinson, n (\%)

levodopa/carbidopa

pramipexole

amantadita

levodopa/carbidopa/ entacapone

biperideno

bromocriptina

selegilina

monoterapia con levodopa/

carbidopa

monoterapia pramipexole

monoterapia levodopa/carbidopa/ entacapone

antecedente manejo quirúrgico

para EP

*EPOC enfermedad pulmonar obstructiva crónica referida por paciente. $\dagger$ SAHOS, síndrome de apnea hipopnea obstructiva del sueño referido por paciente. 


\section{Tabla 3. Distribución dominios y frecuencias de SNM}

\section{Tracto gastrointestinal}

estreñimiento

tenesmo rectal (sensación de no vaciar completo al

defecar)

ageusia, anosmia (pérdida o alteración en percepción

disfagia (dificultad para tragar sólidos o líquidos)

sialorrea (babeo)

vómito o náuse

incontinencia fecal

\section{Tracto Urinario}

urgencia urinaria (sensación urgente de orinar)

nicturia (necesidad de levantarse en la noche a orinar)

\section{Dolor}

dolor sin causa aparente

\section{Misceláneos}

caidas

cambio de peso

diplopia (visión doble)

\section{Apatíal atención / memoria}

memoria (problemas recordar cosas pasadas) 34

apatía (pérdida del interés en lo que pasa alrededor o

en sus cosas)

concentracion (dificultad para concentrarse o mantener la atención)

depresión/ ansiedad/ anhedonia

depresión (sentirse triste o bajo ánimo )

ansiedad (sensación de ansiedad o miedo)

\section{Alucinaciones}

alucinaciones (ver u oír cosas que no existen)

deluciones (creer que le pasan cosas que no son ciertas)

Función sexual

pérdida o aumento de la líbido

dificultades para relación sexual

\section{Sueño y fatiga}

sensación desagradable en piernas por la noche

sensación de mareo o debilidad

insomnio (dificultad para mantenerse dormido)

sueños intensos o pesadillas

hablar o moverse durante el sueño

somnolencia diurna (incapacidad para mantenerse

despierto)

\section{Cardiovascular}

sudoración excesiva

hinchazón de las piernas

(32.7)

() Vocabulario utilizado en el cuestionario aplicado a la población; tsensación desagradable en las piernas por la noche o cuando descansa y sensación de que necesita moverlas.

\begin{tabular}{|c|c|c|}
\hline Tiempo de evolución & $\leq 5$ años & $\begin{array}{l}>5 \\
\text { años }\end{array}$ \\
\hline Sintomas no motores & (\%) & $(\%)$ \\
\hline Urgencia urinaria & 75,4 & 77,7 \\
\hline Sensación desagradable en piernas por la noche & 71,3 & 62,9 \\
\hline Sentirse triste o con bajo ánimo & 67,3 & 81,4 \\
\hline Sensación de mareo o debilidad & 64,2 & 66,6 \\
\hline Nicturia & 64,2 & 77,7 \\
\hline Dolor sin causa aparente & 60,7 & 48,1 \\
\hline Problemas para recordar cosas pasadas & 60,7 & 62,9 \\
\hline Pérdida del interés en sus actividades & 50 & 62,9 \\
\hline Dificultad para concentrarse & 50 & 55,5 \\
\hline Sensación de ansiedad o miedo & 50 & 48,1 \\
\hline Sudoración excesiva & 50 & 37 \\
\hline Pérdida o aumento del interés por la líbido & 46,4 & 40,7 \\
\hline Dificultades para relación sexual & 42,8 & 25,9 \\
\hline Sueños intensos o pesadillas & 42,8 & 48,1 \\
\hline Cambio de peso & 42,8 & 33,3 \\
\hline Insomnio & 39,2 & 62,9 \\
\hline Ageusia, anosmia & 39,2 & 40,7 \\
\hline Caidas & 35,7 & 40,7 \\
\hline Hablar o moverse durante el sueño & 32,1 & 40,7 \\
\hline Hinchazón de las piernas & 32,1 & 33,3 \\
\hline Somnolencia diurna & 28,5 & 40,7 \\
\hline Diplopía & 28,5 & 29,6 \\
\hline Disfagia & 28,5 & 37 \\
\hline Sialorrea & 25 & 29,6 \\
\hline Alucinaciones visuales, auditivas & 25 & 25,9 \\
\hline Deluciones & 17,8 & 14,8 \\
\hline Vómito o náusea & 14,2 & 25,9 \\
\hline Estreñimiento & 46,4 & 48,1 \\
\hline Tenesmo rectal & 39,2 & 48,1 \\
\hline Incontinencia fecal & 10,7 & 11.1 \\
\hline
\end{tabular}

\section{Discusión}

Este es el primer estudio sobre SNM en EP en el Hospital de San José, en el que se ha logrado caracterizar una población de pacientes y registrar la frecuencia de aparición. Los SNM incluyen un amplio espectro, desde la alteración en la función urinaria hasta alucinaciones y varios de ellos, si no todos, pueden estar presentes en el mismo paciente, haciéndose más 
frecuentes a medida que avanza en su evolución la enfermedad. En poblaciones con tiempo mayor de siete años se puede ver hasta en un $88 \%$ la presencia de los mismos. Muchos de estos síntomas no son comentados al médico tratante a menos que se indague con preguntas puntuales, lo que conlleva a la necesidad de su búsqueda mediante un instrumento estandarizado, teniendo en cuenta que deben explorarse en la consulta diaria para brindar manejo oportuno.

Nuestros datos contrastan con lo descrito por Chaudhuri y col. ${ }^{3,9}$ donde el $1.6 \%$ negaron haber presentado SNM. En el grupo de pacientes objeto de nuestro estudio todos presentaron por lo menos un SNM sin importar el tiempo de evolución. Llama la atención que en el estudio piloto The NMS Quest Study realizado en el 2006, ${ }^{9}$ la frecuencia de presentación de los SNM es mayor en nuestra población, con similar comportamiento en la distribución por dominios.

Las alteraciones de los pacientes con duración de la enfermedad mayor de cinco años en orden de frecuencia, como síntomas principales fueron: urgencia urinaria, sentirse triste, alteración de memoria, apatía, insomnio, siendo este ultimo uno de los que presentó mayor registro a medida que avanzaba la enfermedad, que corresponde a lo descrito en la literatura internacional donde las manifestaciones no motores se hacen más frecuentes a medida que evoluciona la enfermedad. ${ }^{9}$

En contraste con lo anterior, al comparar la presentación de los síntomas entre los grupos, a mayor tiempo de evolución hay una disminución en la presentación de sensación desagradable en las piernas por la noche, dolor sin causa aparente, sudoración excesiva, dificultades para la relación sexual y cambio de peso. Algunos de estos resultados bajos pueden atribuirse a dificultades a la hora de interpretar el instrumento en lo relativo a la esfera sexual, pues si el paciente no ha intentado tener relaciones sexuales puede asumir que no presenta problemas, resultando en una menor frecuencia en la enfermedad avanzada. De manera similar al interrogar cambio de peso, se evalúa considerando el período de las cuatro semanas previas cuando puede no percibir fluctuaciones, generando de nuevo una disminución del registro con relación al tiempo de evolución de la enfermedad.

Al evaluar los subgrupos de acuerdo con la terapia farmacológica formulada y la presentación de SNM, si recibía levodopa/carbidopa en monoterapia o en asociación con pramipexol, la urgencia urinaria fue más frecuente en el grupo de politerapia, como también sucedió con la nicturia. Ocurrió lo contrario con la presencia de piernas inquietas en donde los pacientes con politerapia mostraron un descenso en la frecuencia de presentación de este síntoma, resultado que se apoya con lo descrito en la literatura en donde se ha demostrado un papel terapéutico del pramipexol en el tratamiento de esta entidad. ${ }^{18}$ Así mismo, con la terapia combinada hubo menor número de casos con náusea y vómito probablemente debido a la menor dosis de la levodopa/carbidopa requerida cuando se prescribe con fundamento y racionalidad clínica. La reducción de eventos adversos con terapia combinada podría impacta en forma favorable la calidad de vida.

No hubo cambios importantes en la proporción de síntomas como problemas para recordar las cosas, sensación de mareo, cambios en la líbido y estreñimiento entre los grupos en monoterapia levodoca/carbidopa o la asociación con pramipexol, presentándose en la mitad de los casos independiente del esquema terapéutico.

Dentro de los pacientes que presentaban problemas a la hora de tener relaciones sexuales, $73.6 \%$ sentían tristeza al considerar la influencia de las disfunciones en esta esfera y los cambios en estado anímico. Se encontró en nuestra serie de casos que en aquellos con depresión, el 83\% coexistía con urgencia urinaria, importante para establecer o modificar estrategias terapéuticas y evaluar interrelación.

Síntomas como piernas inquietas y nicturia presentaron igual distribución en $78.5 \%$ del total de pacientes con insomnio, que se explica porque el patrón del sueño tiene causas multifactoriales, en especial al relacionar síntomas del mismo dominio. ${ }^{18}$ A su vez, el insomnio se encontró en $63 \%$ de quienes tenían somnolencia diurna, siendo las alteraciones en el ciclo de sueño frecuentes y demostrando la secuencia donde 
al afectarse una etapa repercute sobre el período de vigilia o sueño siguiente.

El instrumento demostró en nuestra población la presencia de los SNM motores, sin poder medir la severidad de los mismos, ni pretendemos con una serie de casos atribuirlos solo a la enfermedad. Al encontrar estos síntomas es útil utilizar esta escala como una herramienta en los centros de atención, mientras espera su valoración neurológica periódica para posterior profundización con escalas más específicas. Se requieren otros análisis y evaluar comorbilidades y efectos secundarios de medicamentos que puedan explicar la coexistencia de SNM. De cualquier manera, sean originados por la enfermedad o tengan otra causa aparente, estos afectarán aun más la calidad de vida en los pacientes con EP.

\section{Conclusiones}

Los SNM fueron frecuentes en nuestra población, comprometían múltiples dominios, donde sobresalen las alteraciones en sistema autonómico. Las principales manifestaciones se documentaron en relación con el tracto urinario, además alteraciones en el estado anímico y la esfera del sueño. Muchos de ellos coexisten en un mismo individuo y la frecuencia de presentación en su gran mayoría se incrementa a medida que evoluciona en el tiempo la EP. En nuestra consulta se debe indagar sobre estos síntomas para brindar un manejo integral al paciente. Se requieren nuevos estudios en nuestra población que evalúen el impacto de los mismos en la calidad de vida, valoren las comorbilidades y los efectos secundarios de medicamentos que puedan explicar la coexistencia de síntomas en estos pacientes.

\section{Referencias}

1. Braak H, Del TK. Invited Article: Nervous system pathology in sporadic Parkinson disease. Neurology. 2008 May 13;70(20):1916-25.

2. Nebe A, Ebersbach G. Selective diplopia in Parkinson's disease: a special subtype of visual hallucination?. Mov Disord. 2007 Jun $15 ; 22(8): 1175-8$.
3. Argandoña-Palacios L, Perona-Moratalla AB, Hernandez-Fernandez F, DiazMaroto I, Garcia-Munozguren S. [Non-motor disorders in Parkinson's disease: introduction and general features]. Rev Neurol. 2010 Feb 8; 50 Suppl 2:S1-S5.

4. Pradilla AG, Vesga AB, Leon-Sarmiento FE. [National neuroepidemiological study in Colombia (EPINEURO)]. Rev Panam Salud Publica. 2003 Aug; 14(2): 104-11

5. Braak H, Del TK, Rub U, de Vos RA, Jansen Steur EN, Braak E. Staging of brain pathology related to sporadic Parkinson's disease. Neurobiol Aging. 2003 Mar;24(2): 197-211.

6. Marco-Llorente J, Rojo-Martinez E. [Other non-motor disorders in Parkinson's disease]. Rev Neurol. 2010 Feb 8; 50 Suppl 2:S75-S83.

7. Martinez-Martin P, Rodriguez-Blazquez C, Abe K, Bhattacharyya KB, Bloem $\mathrm{BR}$, Carod-Artal FJ, et al. International study on the psychometric attributes of the non-motor symptoms scale in Parkinson disease. Neurology. 2009 Nov 10; 73(19):1584-91.

8. Calabresi P, Picconi B, Parnetti L, Di FM. A convergent model for cognitive dysfunctions in Parkinson's disease: the critical dopamine-acetylcholine synaptic balance. Lancet Neurol. 2006 Nov; 5(11):974-83.

9. Chaudhuri KR, Martinez-Martin P, Schapira AH, Stocchi F, Sethi K, Odin P, et al. International multicenter pilot study of the first comprehensive self-completed nonmotor symptoms questionnaire for Parkinson's disease: the NMSQuest study. Mov Disord. 2006 Jul; 21(7):916-23.

10. Chaudhuri KR, Martinez-Martin P, Brown RG, Sethi K, Stocchi F, Odin P, et al. The metric properties of a novel non-motor symptoms scale for Parkinson's disease: results from an international pilot study. Mov Disord. 2007 Oct 15; 22(13): 1901-11.

11. Chaudhuri KR, Prieto-Jurcynska C, Naidu Y, Mitra T, Frades-Payo B, Tluk S, et al. The nondeclaration of nonmotor symptoms of Parkinson's disease to health care professionals: an international study using the nonmotor symptoms questionnaire. Mov Disord. 2010 Apr 30; 25(6):697-701.

12. Dawson TM, Dawson VL. Rare genetic mutations shed light on the pathogenesis of Parkinson disease. J Clin Invest. 2003 Jan;1 11(2):145-51.

13. Erro ME, Moreno MP, Zandio B. [Pathophysiological bases of the non-motor symptoms in Parkinson's disease]. Rev Neurol. 2010 Feb 8; 50 Suppl 2:S7-13.

14. Forjaz MJ, Frades-Payo B, Martinez-Martin P. [The current state of the art concerning quality of life in Parkinson's disease: II. Determining and associated factors]. Rev Neurol. 2009 Dec 16; 49(12):655-60.

15. Gonzalez-Fernandez J, Prieto-Albin R, Velasco-Palacios L, Jorge-Roldan S, Cubo-Delgado E. [Digestivedisorders in Parkinson'sdisease: dysphagia and sialorrhea]. Rev Neurol. 2010 Feb 8; 50 Suppl 2:S51-S54.

16. Racette BA, Gokden MS, Tychsen LS, Perlmutter JS. Convergence insufficiency in idiopathic Parkinson's disease responsive to levodopa. Strabismus. 1999 Sep;7(3): 169-74

17. Mondragon-Rezola E, Arratibel-Echarren I, Ruiz-Martinez J, Marti-Masso JF. [Sleep disorders in Parkinson's disease: insomnia and sleep fragmentation, daytime hypersomnia, alterations to the circadian rhythm and sleep apnea syndrome]. Rev Neurol. 2010 Feb 8;50 Supp.1 2:S21-S26.

18. Jauregui-Barrutia A, Tijero-Merino B, Gomez-Esteban JC, Zarranz JJ. [Sleep disorders in Parkinson's disease: REM sleep behaviour disorder and restless legs syndrome]. Rev Neurol. 2010 Feb 8;50 Suppl 2:S15-S19.

19. Jellinger KA. A critical reappraisal of current staging of Lewy-related pathology in human brain. Acta Neuropathol. $2008 \mathrm{Jul} ; 116(1): 1-16$.

20. Vazquez-Saanchez F, Rodriguez-Martinez E, Ares-Luque A. [Urinary disorders, sexual dysfunction and hypersexuality in Parkinson's disease]. Rev Neurol. 2010 Feb 8; 50 Suppl 2:S27-S31.

21. Zesiewicz TA, Sullivan KL, Arnulf I, Chaudhuri KR, Morgan JC, Gronseth GS, et al. Practice Parameter: treatment of nonmotor symptoms of Parkinson disease: report of the Quality Standards Subcommittee of the American Academy of Neurology. Neurology. 2010 Mar 16;74(11):924-31. 